\title{
Search for Neutrinoless Double- $\beta$ Decay with the Complete EXO-200 Dataset
}

G. Anton, ${ }^{1}$ I. Badhrees, ${ }^{2, a}$ P. S. Barbeau, ${ }^{3}$ D. Beck, ${ }^{4}$ V. Belov, ${ }^{5}$ T. Bhatta, ${ }^{6}$ M. Breidenbach, ${ }^{7}$ T. Brunner,${ }^{8,9}$ G. F. Cao, ${ }^{10}$ W. R. Cen, ${ }^{10}$ C. Chambers, ${ }^{11, b}$ B. Cleveland, ${ }^{12, \mathrm{c}}$ M. Coon, ${ }^{4}$ A. Craycraft, ${ }^{11}$ T. Daniels, ${ }^{13}$ M. Danilov, ${ }^{5, \mathrm{~d}}$ L. Darroch, ${ }^{8}$ S. J. Daugherty, ${ }^{14}$ J. Davis, ${ }^{7}$ S. Delaquis, ${ }^{7, *}$ A. Der Mesrobian-Kabakian, ${ }^{12}$ R. DeVoe, ${ }^{15}$ J. Dilling, ${ }^{9}$ A. Dolgolenko, ${ }^{5}$ M. J. Dolinski, ${ }^{16}$ J. Echevers, ${ }^{4}$ W. Fairbank, Jr., ${ }^{11}$ D. Fairbank, ${ }^{11}$ J. Farine, ${ }^{12}$ S. Feyzbakhsh, ${ }^{17}$ P. Fierlinger, ${ }^{18}$ D. Fudenberg, ${ }^{15}$ P. Gautam, ${ }^{16}$ R. Gornea, ${ }^{2,9}$ G. Gratta,${ }^{15}$ C. Hall, ${ }^{19}$ E. V. Hansen, ${ }^{16}$ J. Hoessl, ${ }^{1}$ P. Hufschmidt,${ }^{1}$ M. Hughes,${ }^{20}$ A. Iverson, ${ }^{11}$ A. Jamil, ${ }^{21}$ C. Jessiman, ${ }^{2}$ M. J. Jewell, ${ }^{15}$ A. Johnson, ${ }^{7}$ A. Karelin, ${ }^{5}$ L. J. Kaufman, ${ }^{7, e}$ T. Koffas, ${ }^{2}$ R. Krücken, ${ }^{9}$ A. Kuchenkov, ${ }^{5}$ K. S. Kumar, ${ }^{22, f}$ Y. Lan, ${ }^{9}$ A. Larson, ${ }^{6}$ B. G. Lenardo, ${ }^{15}$ D. S. Leonard ${ }^{23}$ G. S. Li $\odot,{ }^{15, \dagger}$ S. Li, ${ }^{4}$ Z. Li, ${ }^{21}$ C. Licciardi, ${ }^{12}$ Y. H. Lin, ${ }^{16}$ R. MacLellan, ${ }^{6}$ T. McElroy, ${ }^{8}$ T. Michel, ${ }^{1}$ B. Mong, ${ }^{7}$ D. C. Moore, ${ }^{21}$ K. Murray, ${ }^{8}$ O. Njoya,${ }^{22}$ O. Nusair, ${ }^{20}$ A. Odian, ${ }^{7}$ I. Ostrovskiy, ${ }^{20}$ A. Piepke, ${ }^{20}$ A. Pocar, ${ }^{17}$ F. Retière, ${ }^{9}$ A. L. Robinson, ${ }^{12}$ P. C. Rowson, ${ }^{7}$ D. Ruddell, ${ }^{13}$ J. Runge, ${ }^{3}$ S. Schmidt, ${ }^{1}$ D. Sinclair, ${ }^{2,9}$ A. K. Soma,${ }^{20}$ V. Stekhanov, ${ }^{5}$ M. Tarka,${ }^{17}$ J. Todd, ${ }^{11}$ T. Tolba, ${ }^{10}$ T. I. Totev, ${ }^{8}$ B. Veenstra, ${ }^{2}$ V. Veeraraghavan, ${ }^{20}$ P. Vogel,${ }^{24}$ J.-L. Vuilleumier ${ }^{25}$ M. Wagenpfeil,,${ }^{1}$ J. Watkins, ${ }^{2}$ M. Weber,${ }^{15}$ L. J. Wen, ${ }^{10}$ U. Wichoski, ${ }^{12}$ G. Wrede, ${ }^{1}$ S. X. Wu,${ }^{15}$ Q. Xia, ${ }^{21}$ D. R. Yahne, ${ }^{11}$ L. Yang, ${ }^{4}$ Y.-R. Yen, ${ }^{16}$ O. Ya. Zeldovich,,${ }^{5}$ and T. Ziegler ${ }^{1}$

(EXO-200 Collaboration)

\footnotetext{
${ }^{1}$ Erlangen Centre for Astroparticle Physics, Friedrich-Alexander-University Erlangen-Nürnberg, Erlangen 91058, Germany

${ }^{2}$ Physics Department, Carleton University, Ottawa, Ontario K1S 5B6, Canada

${ }^{3}$ Department of Physics, Duke University, and Triangle Universities Nuclear Laboratory, Durham, North Carolina 27708, USA

${ }^{4}$ Physics Department, University of Illinois, Urbana-Champaign, Illinois 61801, USA

${ }^{5}$ Institute for Theoretical and Experimental Physics named by A.I. Alikhanov of National Research Center "Kurchatov Institute," 117218 Moscow, Russia

${ }^{6}$ Department of Physics, University of South Dakota, Vermillion, South Dakota 57069, USA

${ }^{7}$ SLAC National Accelerator Laboratory, Menlo Park, California 94025, USA

${ }^{8}$ Physics Department, McGill University, Montreal H3A 2T8, Quebec, Canada

${ }^{9}$ TRIUMF, Vancouver, British Columbia V6T 2A3, Canada

${ }^{10}$ Institute of High Energy Physics, Beijing 100049, China

${ }^{11}$ Physics Department, Colorado State University, Fort Collins, Colorado 80523, USA

${ }^{12}$ Department of Physics, Laurentian University, Sudbury, Ontario P3E 2C6, Canada

${ }^{13}$ Department of Physics and Physical Oceanography, University of North Carolina at Wilmington, Wilmington, North Carolina 28403, USA

${ }^{14}$ Physics Department and CEEM, Indiana University, Bloomington, Indiana 47405, USA

${ }^{15}$ Physics Department, Stanford University, Stanford, California 94305, USA

${ }^{16}$ Department of Physics, Drexel University, Philadelphia, Pennsylvania 19104, USA

${ }^{17}$ Amherst Center for Fundamental Interactions and Physics Department, University of Massachusetts, Amherst, Massachusetts 01003, USA

${ }^{18}$ Physik Department and Excellence Cluster Universe, Technische Universität München, Garching 80805, Germany

${ }^{19}$ Physics Department, University of Maryland, College Park, Maryland 20742, USA

${ }^{20}$ Department of Physics and Astronomy, University of Alabama, Tuscaloosa, Alabama 35487, USA

${ }^{21}$ Wright Laboratory, Department of Physics, Yale University, New Haven, Connecticut 06511, USA

${ }^{22}$ Department of Physics and Astronomy, Stony Brook University, SUNY, Stony Brook, New York 11794, USA

${ }^{23}$ IBS Center for Underground Physics, Daejeon 34126, Korea

${ }^{24}$ Kellogg Lab, Caltech, Pasadena, California 91125, USA

${ }^{25}$ LHEP, Albert Einstein Center, University of Bern, Bern CH-3012, Switzerland
}

(Received 10 June 2019; revised manuscript received 30 July 2019; published 18 October 2019)

A search for neutrinoless double- $\beta$ decay $(0 \nu \beta \beta)$ in ${ }^{136} \mathrm{Xe}$ is performed with the full EXO-200 dataset using a deep neural network to discriminate between $0 \nu \beta \beta$ and background events. Relative to previous

\footnotetext{
Published by the American Physical Society under the terms of the Creative Commons Attribution 4.0 International license. Further distribution of this work must maintain attribution to the author(s) and the published article's title, journal citation, and DOI. Funded by $S C O A P^{3}$.
} 
analyses, the signal detection efficiency has been raised from $80.8 \%$ to $96.4 \pm 3.0 \%$, and the energy resolution of the detector at the $Q$ value of ${ }^{136} \mathrm{Xe} 0 \nu \beta \beta$ has been improved from $\sigma / E=1.23 \%$ to $1.15 \pm$ $0.02 \%$ with the upgraded detector. Accounting for the new data, the median $90 \%$ confidence level $0 \nu \beta \beta$ half-life sensitivity for this analysis is $5.0 \times 10^{25} \mathrm{yr}$ with a total ${ }^{136} \mathrm{Xe}$ exposure of $234.1 \mathrm{~kg}$ yr. No statistically significant evidence for $0 \nu \beta \beta$ is observed, leading to a lower limit on the $0 \nu \beta \beta$ half-life of $3.5 \times 10^{25} \mathrm{yr}$ at the $90 \%$ confidence level.

DOI: 10.1103/PhysRevLett.123.161802

Double- $\beta$ decay is a second-order weak transition in which two neutrons simultaneously decay into two protons. Although the mode with emission of two electrons and two antineutrinos $(2 \nu \beta \beta)$ has been observed in several nuclides in which single- $\beta$ decay was suppressed [1], the hypothetical neutrinoless mode $(0 \nu \beta \beta)$ [2] is yet to be discovered. The search for $0 \nu \beta \beta$ is recognized as the most sensitive probe for the Majorana nature of neutrinos [3-6]. Its observation would provide direct evidence for a beyondthe-Standard-Model process that violates lepton number conservation as well as helps constrain the absolute mass scale of neutrinos [7].

Recent experiments probing a number of nuclides [8-12] have set lower limits on the $0 \nu \beta \beta$ half-life with sensitivities in the range of $10^{25}-10^{26} \mathrm{yr}$ at a $90 \%$ confidence level (CL). Exploiting the advantages of a liquid xenon (LXe) cylindrical time projection chamber (TPC) filled with LXe enriched to $80.6 \%$ in ${ }^{136} \mathrm{Xe}$ [13], the EXO-200 [14] experiment achieved a sensitivity of $3.7 \times 10^{25} \mathrm{yr}$ with the most recent $0 \nu \beta \beta$ search [8], whereas the most sensitive search to date for the same isotope reached $5.6 \times 10^{25} \mathrm{yr}$ [9]. Here, we report on a search with similar sensitivity to the previous best search.

In December 2018, the EXO-200 experiment completed data taking with the upgraded detector ("Phase II," May 2016 to December 2018) after collecting an exposure similar to that of its first run ("Phase I," September 2011 to February 2014). This Letter reports a search for $0 \nu \beta \beta$ using the full EXO-200 dataset that, after data quality cuts [13], totals $1181.3 \mathrm{~d}$ of live time. This represents an approximately $25 \%$ increase in exposure relative to the previous search [8] that already included nearly half of the Phase II dataset. In addition to the new data acquired between June 2017 and October 2018, this search introduces several analysis developments to optimize the detector sensitivity to $0 \nu \beta \beta$, including the incorporation of a deep neural network (DNN) to discriminate between background and signal events.

In the EXO-200 detector, a common cathode splits the LXe TPC into two drift regions, each with a radius of $\sim 18 \mathrm{~cm}$ and a drift length of $\sim 20 \mathrm{~cm}$. The TPC is enclosed by a radiopure thin-walled copper vessel. The electric field in the drift regions was raised from $380 \mathrm{~V} / \mathrm{cm}$ in Phase I to $567 \mathrm{~V} / \mathrm{cm}$ in Phase II to improve the energy resolution because it was found that, after the detector was restarted, higher voltage values on the cathode were stable.
The ionization produced from interactions in the LXe is read out after being drifted to crossed-wire planes at each anode, whereas the scintillation light produced at the interaction time is collected by arrays of large area avalanche photodiodes (LAAPDs) [15] located behind the wire planes.

The underground location of the experiment, which is the Waste Isolation Pilot Plant (WIPP) near Carlsbad, New Mexico, provides an overburden of $1624_{-21}^{+22} \mathrm{~m}$ of water equivalent [16]. In addition to several layers of passive shielding (including $\sim 50 \mathrm{~cm}$ of HFE-7000 cryofluid [17], $5.4 \mathrm{~cm}$ of copper, and $\sim 25 \mathrm{~cm}$ of lead in all directions [14]), an active muon veto system with scintillator panels on four sides allows prompt identification of $>94 \%$ of the cosmic ray muons passing through the TPC. This system is also used in this analysis to reject background events arising from cosmogenically produced ${ }^{137} \mathrm{Xe}$, which primarily decays via $\beta$ emission with a half-life of $3.82 \mathrm{~min}[8,18]$.

Each TPC event is reconstructed by grouping charge and light signals into individual energy deposits. Ionization signals measured by two wire planes, an induction plane (V wires) and a collection plane (U wires), provide information about coordinates $x$ and $y$ perpendicular to the drift field. The $z$ position, along the drift direction, is obtained from the time delay between the prompt light and the delayed charge signals together with the measured electron drift velocity [19]. Events reconstructed with single and multiple energy deposit(s) are referred to as "single site" (SS) and "multisite" (MS). $0 \nu \beta \beta$ events are predominantly SS, whereas backgrounds are mostly MS. Although the main procedures for spatial reconstruction are the same as in previous searches $[8,13,20]$, the $0 \nu \beta \beta$ detection efficiency has been raised to $97.8 \pm 3.0 \%$ $(96.4 \pm 3.0 \%)$ in Phase I (Phase II) from $82.4 \pm 3.0 \%$ $(80.8 \pm 2.9 \%)$ [8] by relaxing two selection criteria. First, the time required for events to be separated from all other reconstructed events has been reduced from $>1 \mathrm{~s}$ to $>0.1 \mathrm{~s}$. This time cut is still at least two orders of magnitude longer than expected from typical time-correlated backgrounds seen in the detector [16,21], whereas the $0 \nu \beta \beta$ efficiency loss due to accidental coincidence is reduced from $7 \%$ to $0.5 \%$. Second, the search presented here includes events containing deposits without a detected V-wire signal if these deposits contribute $<40 \%$ of the total event energy, which were removed in the previous analyses. Because of the higher energy threshold for signal detection 
on the $\mathrm{V}$ wires $(\sim 200 \mathrm{keV})$ versus the $\mathrm{U}$ wires $(\sim 90 \mathrm{keV})$, a significant number of events with small energy deposits are well reconstructed by the $\mathrm{U}$ wires but incompletely on the $\mathrm{V}$ wires, resulting in events with full $z$ reconstruction but incomplete $x y$ reconstruction for smaller energy deposits. Relaxing this 3D cut criterion only recovers MS events and retrieves almost all potential $0 \nu \beta \beta$ events with incomplete $x y$ reconstruction due to small, separated energy deposits from bremsstrahlung. Although $0 \nu \beta \beta$ primarily induces SS events, the smaller fraction of MS $0 \nu \beta \beta$ events can be distinguished from the dominant $\gamma$ backgrounds using a discriminator for MS events (described below), resulting in an enhancement in the $0 \nu \beta \beta$ half-life sensitivity.

Events within the fiducial volume $(\mathrm{FV})$ are required to lie within a hexagon in the $x y$ plane with an apothem of $162 \mathrm{~mm}$. They are further required to be more than $10 \mathrm{~mm}$ away from the cylindrical polytetrafluoroethylene (PTFE) reflector, as well as the cathode and the $\mathrm{V}$-wire planes. This FV contains $3.31 \times 10^{26}$ atoms of ${ }^{136} \mathrm{Xe}$, with an equivalent mass of $74.7 \mathrm{~kg}$. Although the incomplete $x y$-matched energy deposits may fall outside the FV, this effect is determined by detector simulations to have a negligible effect on the estimated detection efficiency due to the energy requirements imposed on these events. The ${ }^{136} \mathrm{Xe}$ exposure of the entire dataset after data quality cuts and accounting for live time loss due to vetoing events coincident with the muon veto is $234.1 \mathrm{~kg} \mathrm{yr}$, or 1727.5 mol yr, with 117.4 (116.7) kg yr in Phase I (Phase II).

The detector response to $0 \nu \beta \beta$ decays and background interactions is modeled by a detailed Monte Carlo (MC) simulation based on GEANT4 [22]. This MC simulation models the energy deposits produced by interactions in the $\mathrm{LXe}$, and then it propagates the ionization through the detector to produce waveforms associated to readout channels. These simulated waveforms are input to the same reconstruction and analysis framework used for data waveforms. Calibration data with external $\gamma$ sources located 9 (11) $\mathrm{cm}$ away from the FV at set positions around the cathode (behind the anodes) [13] were regularly taken to validate the analysis.

After the previous EXO-200 $0 \nu \beta \beta$ search [8], a small fraction of the observed candidate events presented light-tocharge ratios that were not fully consistent with their expected distributions. Using calibration and $2 \nu \beta \beta$ data, the distribution of the light-to-charge ratio is measured and found to be approximately Gaussian around the mean ratio. While keeping the maximal search sensitivity, a cut is imposed requiring that events are within $2.5 \sigma$ of the mean of the distribution. This improves the previous cut [13], primarily aimed at removing $\alpha$ decays, by also removing poorly reconstructed $\beta$ and $\gamma$ events with an anomalous light-to-charge ratio. All systematic errors associated with the signal detection efficiency are summarized in Table I.

The reconstructed energy is determined by combining the anticorrelated charge and light signals [23] to optimize the
TABLE I. Summary of systematic error contributions.

\begin{tabular}{lcc}
\hline \hline Source & Phase I (\%) & Phase II (\%) \\
\hline Background errors & & \\
Spectral shape agreement & 5.8 & 4.4 \\
Background model & 4.0 & 4.6 \\
Other [8] & 1.5 & 1.2 \\
Total error & 7.1 & 6.5 \\
Signal detection efficiency & & \\
Fiducial volume & 2.8 & 2.6 \\
Partial 3D cut & $<0.4$ & $<0.4$ \\
Light-to-charge ratio & 0.9 & 0.9 \\
Denoising misreconstructed & $\ldots$ & 1.0 \\
Other [13] & $<1.0$ & $<1.0$ \\
Total error & 3.1 & 3.1 \\
\hline \hline
\end{tabular}

resolution at the $0 \nu \beta \beta$ decay energy of $Q_{\beta \beta}=2457.83 \pm$ $0.37 \mathrm{keV}$ [24]. An offline denoising algorithm [25], previously introduced to account for excess APD readout noise observed in Phase I, has been further optimized with measurements of the light response of the detector and adapted for Phase II data. In addition, a proper modeling of mixed signals from the induced and collected charge in wires is introduced to the signal finder in the event reconstruction process. The resulting energy measurement shows good spectral agreement between the data and the simulation for SS and MS events using ${ }^{228} \mathrm{Th},{ }^{226} \mathrm{Ra}$, and ${ }^{60}$ Co calibration sources, as shown in Fig. 1. The electronics upgrade carried out before Phase II data taking resulted in substantially improved resolution and stability, as illustrated in Fig. 2. The average detector resolution is determined by uniformly weighting all calibration data from several positions and accounting for the detector live time. The averages for Phase I and Phase II are $\sigma / E\left(Q_{\beta \beta}\right)=$ $1.35 \pm 0.09 \%$ and $1.15 \pm 0.02 \%$, respectively.

All data, including those previously reported, were blinded to hide all candidate $0 \nu \beta \beta$ SS events having energy

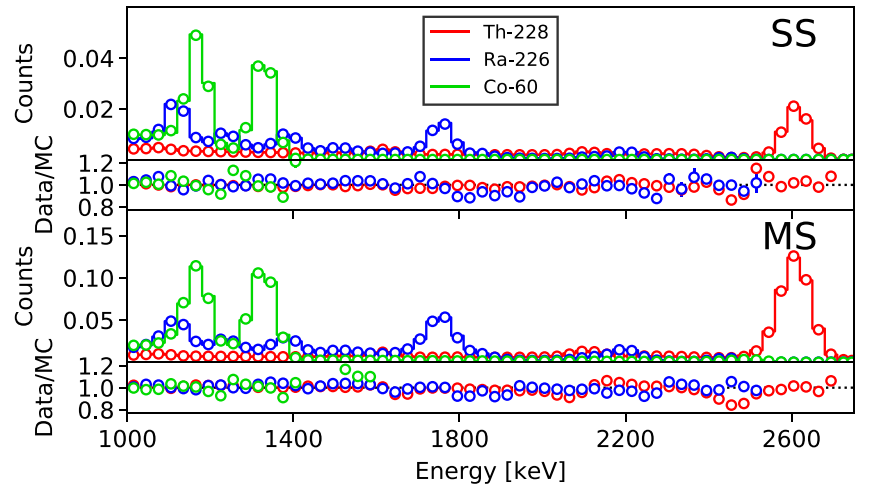

FIG. 1. Comparison of energy distributions in data (circles) and MC (lines) for SS (top half) and MS (bottom half) events from calibration sources positioned near the cathode. 


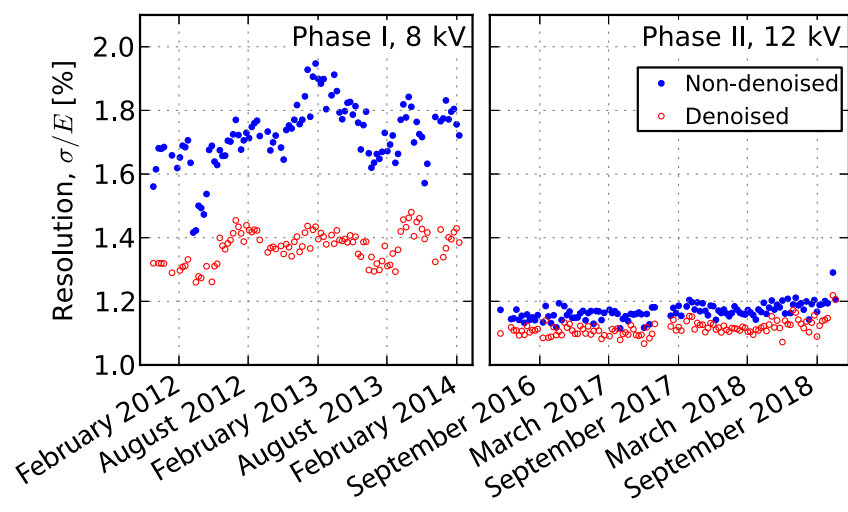

FIG. 2. Energy resolution of SS events measured using a ${ }^{228} \mathrm{Th}$ calibration source deployed to a position near the cathode. The effect of the denoising algorithm and weekly variation of the resolution at the $2615 \mathrm{keV}^{208} \mathrm{Tl} \gamma$ line are shown for both Phase I and Phase II. The resolution worsened slightly after a xenon recovery in July 2017 due to a power outage. A degraded resolution due to an increase of excess noise is visible in the last weeks of Phase II.

within $Q_{\beta \beta} \pm 2 \sigma$. No information about such events is used in the development of the techniques for this analysis. New background discriminators are studied to optimize the sensitivity of this search while minimizing the systematic errors. The search for $0 \nu \beta \beta$ is performed with a simultaneous maximum-likelihood (ML) fit to the SS and MS energy spectra, with the discriminators added as additional fit dimensions. Although Phase I and Phase II are fit independently and then combined by summing their individual profile likelihoods for various signal hypotheses, both use the same background model developed in [8] composed of decays originating in the detector and surrounding materials. Systematic errors are included in the ML fit as nuisance parameters constrained by normal distributions. The median 90\% CL sensitivity is estimated using toy datasets (simulated trial experiments) generated from the MC probability density functions (PDFs) of the background model.

The primary topological discrimination of backgrounds is the SS and MS event classification. Figure 3 shows the agreement between the source calibration data and $\mathrm{MC}$ for the "SS fraction": SS/(SS + MS). Because the relaxed 3D cut recovers MS events, the SS fraction near $Q_{\beta \beta}$ is lowered from $24 \%(23 \%)$ to $12 \%$ (14\%) for the ${ }^{228} \mathrm{Th}\left({ }^{226} \mathrm{Ra}\right)$ source as compared to previous searches. Systematic errors related to the SS fractions are determined by comparisons between the data and MC. Taking into account different calibration sources at various positions, these systematics are evaluated to be $5.8 \%$ (4.6\%) for Phase I (Phase II).

Motivated by the results in [26], this analysis introduces a new discriminator for SS and MS events using a DNN that relies on the waveforms of U-wire signals and is found to outperform the searches in $[8,20]$. The training inputs for the DNN are gray scale images built by arranging

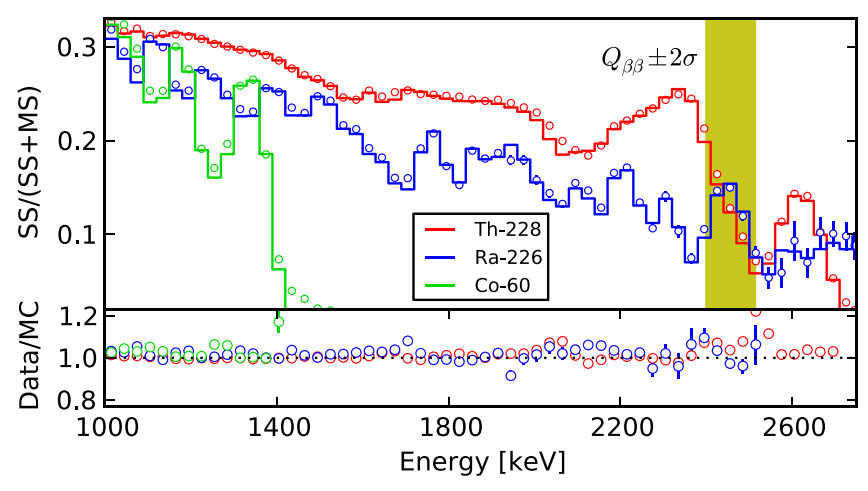

FIG. 3. SS fractions for MC (lines) and data (circles) in Phase II using calibration sources positioned near the cathode.

neighboring channels next to each other and encoding the amplitudes of U-wire waveforms as pixel values. The training data are produced in a MC simulation for two classes of events in equal weights: background-like events composed of $\gamma$ events with uniform energy distribution between 1000 and $3000 \mathrm{keV}$, and signal-like $0 \nu \beta \beta$ events with a random decay energy restricted to the same energy limits. The locations of the simulated interactions of both types are drawn uniformly from the detector volume to focus discrimination only on the topological event characteristics. This dataset is divided into $90 \%$ for training and $10 \%$ for validation. The DNN architecture is inspired by the Inception architecture proposed by Google [27] and implemented with the Keras library [28] using the Tensorflow back end [29].

The agreement for the DNN discriminator between the data and MC is improved when signals from U-wire waveforms are first identified by the signal finder in the EXO-200 reconstruction framework and then used to regenerate the images. Because there is no spatial dependence in training the DNN for signal- and background-like events, the standoff distance (minimum distance between the event position and the closest material surface excluding the cathode) is incorporated in the search as a third fit dimension for both SS and MS events. Figure 4 shows a comparison of these two discriminators between simulated and observed data distributions for the ${ }^{226} \mathrm{Ra}$ calibration source, as well as for the measured background-subtracted $2 \nu \beta \beta$ distribution. While keeping as much discriminating power as possible, the binning used for each variable is selected to minimize systematic errors arising from imperfections in the MC simulation.

Because the fit cannot resolve the detailed location of backgrounds arising from materials far from the LXe vessel, the ${ }^{238} \mathrm{U},{ }^{232} \mathrm{Th}$, and ${ }^{60} \mathrm{Co}$ contributions from such components are assigned to fewer representative locations. For example, all far ${ }^{238} \mathrm{U}$ are represented by the decays in the air gap between the cryostats and the lead shielding in the background model. To account for the errors introduced by this approximation, ${ }^{238} \mathrm{U}$ simulated in the cryostats is 


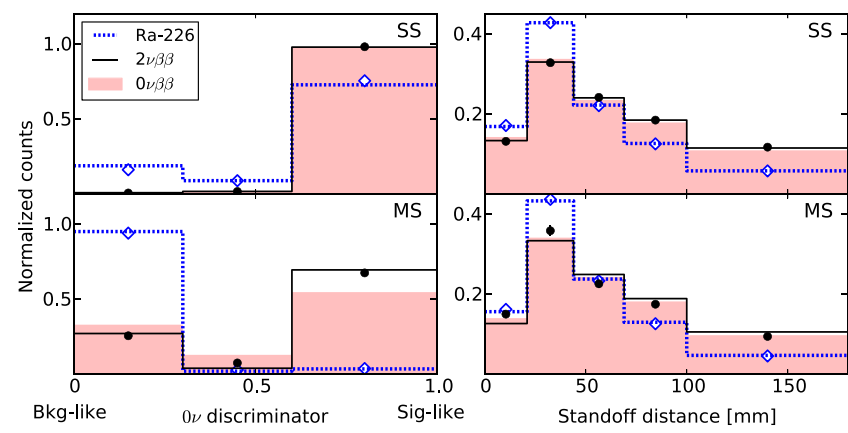

FIG. 4. Comparison between data (dots) and MC (solid and dashed lines) for the DNN $0 \nu \beta \beta$ discriminator (left) and standoff distance (right). Shown are the distributions from the ${ }^{226} \mathrm{Ra}$ calibration source (blue) and the background-subtracted $2 \nu \beta \beta$ spectrum from low background data (black). The simulated distributions for $0 \nu \beta \beta$ events are indicated by the red filled regions. The difference in DNN distributions between $0 \nu \beta \beta$ and $2 \nu \beta \beta$ events in MS is due to the higher rate of bremsstrahlung at higher electron energy.

used to represent all ${ }^{238} \mathrm{U}$ from remote locations. This is taken to represent an extreme deviation from the more realistic case used in the analysis. The resulting change in the expected number of events near $Q_{\beta \beta}$ is taken as the systematic error of the background model. This is evaluated to be $4.0 \%(4.6 \%)$ in Phase I (Phase II) by adding contributions from ${ }^{238} \mathrm{U},{ }^{232} \mathrm{Th}$, and ${ }^{60} \mathrm{Co}$ in quadrature.
In addition, toy studies were used to find the average bias in the expected number of events near $Q_{\beta \beta}$ arising from the measured spectral differences between the data and MC for the energy, the DNN $0 \nu \beta \beta$ discriminator, and the standoff distance. The differences between the data and MC for their distributions obtained with the $\gamma$ calibration sources are used to correct the predicted PDFs, whereas differences in the backgroundsubtracted $2 \nu \beta \beta$ distribution are used for $\beta$-like components. The relative differences between the results with toy datasets generated from the corrected PDFs but fit without this correction are added in quadrature for all contributors and sum to $5.8 \%(4.4 \%)$ in Phase I (Phase II). Table I summarizes the contributions to background errors, including other sources unchanged from previous searches.

The measured rate of radon decays in the LXe is used to constrain the appropriate background components arising from these atoms, as described in [13]. The relative rate of cosmogenically produced backgrounds is also constrained [16]. In addition, a possible difference between the energy scale from $\gamma$ calibration sources $\left(E_{\gamma}\right)$ and from single- or double- $\beta$ decays $\left(E_{\beta}\right)$ is accounted for by a factor $(B)$ that scales the energy of the $\beta$-like components in the ML fit: $E_{\beta}=B E_{\gamma} . B$ is allowed to freely float and is found to be consistent with unity to the subpercent level in both phases.
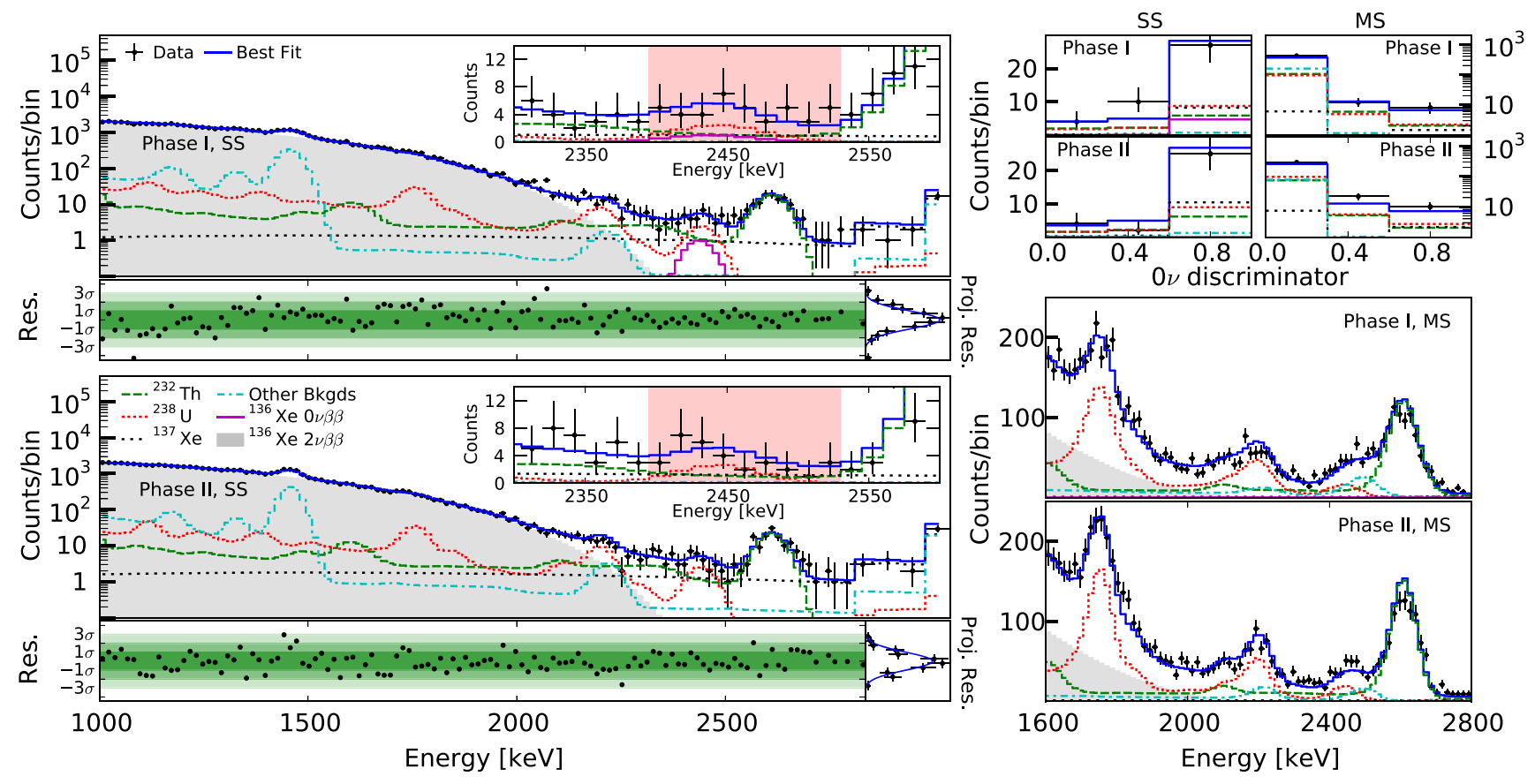

FIG. 5. Best fits to the low background (bkgd) data SS energy spectrum for Phase I (top left) and Phase II (bottom left). The energy bins are 15 and $30 \mathrm{keV}$ below and above $2800 \mathrm{keV}$, respectively. The inset shows an enlarged view around the best-fit value for $Q_{\beta \beta}$. (Top right) Projection of events in the range of 2395 to $2530 \mathrm{keV}$ on the DNN fit dimension for SS and MS events. (Bottom right) MS energy spectra. Best-fit residuals typically follow normal distributions, with small deviations taken into account in the spectral shape systematic errors. 
TABLE II. Best-fit background contributions to $Q_{\beta \beta} \pm 2 \sigma$ versus observed number of events in data.

\begin{tabular}{lccccc}
\hline \hline & ${ }^{238} \mathrm{U}$ & ${ }^{232} \mathrm{Th}$ & ${ }^{137} \mathrm{Xe}$ & Total & Data \\
\hline Phase I & 12.6 & 10.0 & 8.7 & $32.3 \pm 2.3$ & 39 \\
Phase II & 12.0 & 8.2 & 9.3 & $30.9 \pm 2.4$ & 26 \\
\hline \hline
\end{tabular}

The $90 \%$ CL median sensitivity for this $0 \nu \beta \beta$ search with the DNN $0 \nu \beta \beta$ discriminator is evaluated to be $5.0 \times 10^{25} \mathrm{yr}$. The coverage is validated with toy MC studies and found to agree with Wilks's theorem [30,31]. A secondary analysis is performed using a boosted decision tree (BDT) discriminator for MS events, and the BDT discriminator designed in [8] for SS events as the second fit dimension. The BDT for MS is built on variables containing information on the energy fraction of the most energetic deposit, the spatial spread among deposits, and the number of deposits. The BDT analysis provides comparable but slightly worse $(\sim 3 \%)$ sensitivity, suggesting that the discrimination power of the DNN discriminator can be mostly accounted for by careful construction of BDT variables. The DNN analysis was selected as the primary analysis prior to unblinding because it had the best sensitivity.

After unblinding the dataset, the SS candidate events within $Q_{\beta \beta} \pm 2 \sigma$ were examined, which led us to find that one event (originally with energy in this region) was misreconstructed by the denoising algorithm. Its impact on the $0 \nu \beta \beta$ detection efficiency was investigated, and it was found to only affect Phase II with an efficiency loss of $<1.0 \%$. A conservative $1.0 \%$ error was also added to the signal detection efficiency.

ML fits are performed to Phase I and Phase II separately, and the best-fit results are shown in Fig. 5. No statistically significant evidence for $0 \nu \beta \beta$ is observed. The best-fit background contributions in counts to $Q_{\beta \beta} \pm 2 \sigma$ are shown in Table II. The rates normalized over the total fiducial $\mathrm{Xe}$ mass, including all isotopes, are $(1.7 \pm 0.2) \times 10^{-3}$ and $(1.9 \pm 0.2) \times 10^{-3} \mathrm{~kg}^{-1} \mathrm{yr}^{-1} \mathrm{keV}^{-1}$ for Phase I and Phase II, respectively. The lower limit on the ${ }^{136} \mathrm{Xe} 0 \nu \beta \beta$ half-life is derived by profiling over all nuisance parameters, and it results in $T_{1 / 2}>1.7 \times 10^{25} \mathrm{yr}\left(T_{1 / 2}>4.3 \times 10^{25} \mathrm{yr}\right)$ at 90\% CL in Phase I (Phase II), whereas the combined limit is $T_{1 / 2}>3.5 \times 10^{25} \mathrm{yr}$. This corresponds to an upper limit on the Majorana neutrino mass of $\left\langle m_{\beta \beta}\right\rangle<(93-286) \mathrm{meV}$ [5] using the nuclear matrix elements of [32-36] and the phase space factor from [37].

EXO-200 has concluded its operations, reaching a sensitivity to the Majorana neutrino mass of $78-239 \mathrm{meV}$, which is similar to the most sensitive searches for $0 \nu \beta \beta$ to date [9-12]. The analysis presented here utilizes a DNN, which maximally makes use of detailed event topology information for background rejection, leading to an $\sim 25 \%$ improvement relative to the sensitivity using only event energy and simple SS and MS discriminators. This performance results from the unique capabilities of a monolithic LXe TPC, which includes good energy resolution, near maximal signal detection efficiency, and strong topological discrimination of backgrounds. This combination holds promise for nEXO $[38,39]$, which is the planned tonnescale successor to EXO-200, designed to achieve a sensitivity to the $0 \nu \beta \beta$ half-life of $\sim 10^{28} \mathrm{yr}$ in ${ }^{136} \mathrm{Xe}$.

EXO-200 is supported by the DOE and NSF in the U.S., the NSERC in Canada, the SNF in Switzerland, the IBS in Korea, the RFBR (18-02-00550) in Russia, the DFG in Germany, and CAS and ISTCP in China. The EXO-200 data analysis and simulation use resources of the National Energy Research Scientific Computing Center (NERSC). We gratefully acknowledge the KARMEN collaboration for supplying the cosmic-ray veto detectors, as well as the WIPP for their hospitality.

\footnotetext{
* Deceased.

Corresponding author.

ligs@stanford.edu
}

${ }^{a}$ Permanent address: King Abdulaziz City for Science and Technology, Riyadh, Saudi Arabia.

${ }^{b}$ Present address: Physics Department, McGill University, Montreal, Quebec, Canada.

${ }^{\mathrm{c}}$ Also at SNOLAB, Sudbury, Ontario, Canada.

${ }^{\mathrm{d}}$ Present address: P. N. Lebedev Physical Institute of the Russian Academy of Sciences, Moscow, Russia.

eAlso at Physics Department and CEEM, Indiana University, Bloomington, Indiana, USA.

${ }^{\mathrm{f}}$ Present address: Physics Department, University of Massachusetts, Amherst, Massachusetts, USA.

[1] M. Tanabashi et al. (Particle Data Group), Phys. Rev. D 98, 030001 (2018).

[2] W. H. Furry, Phys. Rev. 56, 1184 (1939).

[3] W. Rodejohann, Int. J. Mod. Phys. E 20, 1833 (2011).

[4] J. D. Vergados, H. Ejiri, and F. Simkovic, Rep. Prog. Phys. 75, 106301 (2012).

[5] S. Dell'Oro, S. Marcocci, M. Viel, and F. Vissani, Adv. High Energy Phys. 2016, 2162659 (2016).

[6] M. J. Dolinski, A. W. P. Poon, and W. Rodejohann, arXiv: 1902.04097 [Annu. Rev. Nucl. Part. Phys. (to be published)].

[7] J. Engel and J. Menéndez, Rep. Prog. Phys. 80, 046301 (2017).

[8] J. B. Albert et al. (EXO-200 Collaboration), Phys. Rev. Lett. 120, 072701 (2018).

[9] A. Gando et al. (KamLAND-Zen Collaboration), Phys. Rev. Lett. 117, 082503 (2016).

[10] C. E. Aalseth et al. (MAJORANA Collaboration), Phys. Rev. Lett. 120, 132502 (2018).

[11] M. Agostini et al. (GERDA Collaboration), Phys. Rev. Lett. 120, 132503 (2018).

[12] C. Alduino et al. (CUORE Collaboration), Phys. Rev. Lett. 120, 132501 (2018). 
[13] J. B. Albert et al. (EXO-200 Collaboration), Phys. Rev. C 89, 015502 (2014).

[14] M. Auger et al., J. Instrum. 7, P05010 (2012).

[15] R. Neilson et al., Nucl. Instrum. Methods Phys. Res., Sect. A 608, 68 (2009).

[16] J. B. Albert et al. (EXO-200 Collaboration), J. Cosmol. Astropart. Phys. 04 (2016) 029.

[17] 3M HFE-7000, https://www.3m.com/3M/en_US/companyus/all-3m-products/ /3M-Novec-7000-Engineered-Fluid/? $\mathrm{N}=5002385+3290667267=$ rud.

[18] G. C. Carlson, W. C. Schick, W. L. Talbert, and F. K. Wohn, Nucl. Phys. A125, 267 (1969).

[19] J. B. Albert et al. (EXO-200 Collaboration), Phys. Rev. C 95, 025502 (2017).

[20] J. B. Albert et al. (EXO-200 Collaboration), Nature (London) 510, 229 (2014).

[21] J. B. Albert et al. (EXO-200 Collaboration), Phys. Rev. C 92, 015503 (2015).

[22] J. Allison et al., IEEE Trans. Nucl. Sci. 53, 270 (2006).

[23] E. Conti et al., Phys. Rev. B 68, 054201 (2003).

[24] M. Redshaw, E. Wingfield, J. McDaniel, and E. G. Myers, Phys. Rev. Lett. 98, 053003 (2007).

[25] C. G. Davis et al. (EXO-200 Collaboration), J. Instrum. 11, P07015 (2016).
[26] S. Delaquis et al., J. Instrum. 13, P08023 (2018).

[27] C. Szegedy et al., arXiv:1409.4842.

[28] F. Chollet et al., https://keras.io, 2015.

[29] M. Abadi et al., https://www.tensorflow.org, 2015.

[30] S. S. Wilks, Ann. Math. Stat. 9, 60 (1938).

[31] G. Cowan, Statistical Data Analysis (Oxford Science Publications/Clarendon, Oxford, England, 1998).

[32] J. Barea, J. Kotila, and F. Iachello, Phys. Rev. C 91, 034304 (2015).

[33] N. L. Vaquero, T. R. Rodriguez, and J. L. Egido, Phys. Rev. Lett. 111, 142501 (2013).

[34] J. Engel, F. Simkovic, and P. Vogel, Phys. Rev. C 89, 064308 (2014).

[35] J. Menéndez, A. Poves, E. Caurier, and F. Nowacki, Nucl. Phys. A818, 139 (2009).

[36] M. T. Mustonen and J. Engel, Phys. Rev. C 87, 064302 (2013).

[37] J. Kotila and F. Iachello, Phys. Rev. C 85, 034316 (2012).

[38] S. Al Kharusi et al. (nEXO Collaboration), arXiv:1805 .11142 .

[39] J. B. Albert et al. (nEXO Collaboration), Phys. Rev. C 97, 065503 (2018). 